\title{
Driving-induced stability with long-range effects
}

\author{
Urna Basu ${ }^{1}$, Pierre de Buyl ${ }^{2}$, Christian Maes ${ }^{2}$ and Karel NetočnÝ ${ }^{3}$ \\ 1 SISSA - International School for Advanced Studies and INFN, Trieste, Italy \\ 2 Instituut voor Theoretische Fysica, KU Leuven, Belgium \\ 3 Institute of Physics, Academy of Sciences of the Czech Republic, Prague, Czech Republic
}

\begin{abstract}
PACS 05.40.-a - Fluctuation phenomena, random processes, noise, and Brownian motion
PACS 05.20.-y - Classical statistical mechanics

PACS 05.90.+m-Other topics in statistical physics, thermodynamics, and nonlinear dynamical systems
\end{abstract}

\begin{abstract}
We give a sufficient condition under which a steady nonequilibrium driving in the form of a rotational force on medium particles stabilizes an immersed slow probe in the rotation center. We illustrate that scenario for a diffusive medium with an external potential in the shape of a Mexican hat, high around the origin, with a short-range attraction between those medium particles and a heavier probe. For no or small rotation force on the medium particles, the origin is an unstable fixed point for the probe and the precise shape of the Mexican hat at large distances from the origin is irrelevant for the statistical force there. Above a certain rotation threshold however the probe gets stabilized at the origin and more details of the medium-density at large distance start to matter. The focusing effect occurs for different types of differential rotation with rotation threshold only weakly depending on the ambient temperature and is also robust around the quasi-static limit.
\end{abstract}

Stabilizing an otherwise unstable configuration or phase by external action is an important challenge for a range of applications but also for the physical understanding of the robustness of spatio-temporal patterns induced by contact with nonequilibria. Many examples exist for dynamical systems where by using feedback mechanisms one achieves the necessary control or steering. Other examples such as the Kapitsa (inverted) pendulum which is stabilized by a time-dependent external force do not require feedback 1,2. More recent examples are found in the context of stochastic dynamics with time-dependent driving 5. A further step would be to use the steady nonequilibrium character of a medium to achieve such a stabilization, possibly leading to alternative control strategies. The fact that the medium is quasi-stationary is relevant for the occurrence of stable structures in living matter $[3]$ or in collective Hamiltonian dynamics [4].

In the context of statistical forces, one aims at understanding the action of an ensemble of particles on some collective coordinate or probe. Such a force can be derived consistently in equilibrium statistical mechanics as the derivative of the ensemble free energy. In the present letter we study the statistical force on a slow probe from a nonequilibrium medium. While it can be viewed as an application of the formalism that has been introduced in Refs. [6, 7], we concentrate here on the driving-induced stabilization of a fixed point for the probe's dynamics, similarly to Ref. 5] but here for the case of steady driving. For that purpose we consider a class of models in two dimensions with overdamped diffusive particles that are each subject to a rotational force around the origin (shearing condition) and that interact over a short range with a probe. This is a paradigmatic model of interaction between a slow system (the probe) and a fast system (the medium) where the latter is maintained out of equilibrium by steady driving forces. Medium particles are further confined by an external potential taking the shape of a Mexican hat, which can be viewed as mimicking a mean field interaction between the medium particles but that will play no role in what follows. See Fig. 1 for a graphical summary of the situation. The origin is a fixed point for the probe but for attractive coupling with the medium is unstable in equilibrium. We show in the present paper that it acquires stability when increasing the rotational driving in the medium beyond a threshold value. We will establish that stabilization of the probe at the rotation center by computing the effective spring constant, or stiffness, which is obtained from linearizing the 
statistical force on the probe there. Naturally extending an established terminology 8, we call the nonequilibrium contribution to the symmetrized stiffness a nonequilibrium Lamb shift, and we show that it is given in terms of a covariance between excess work functions. That excess work involves the nonequilibrium density globally and not only at the location of the probe, which is a manifestation that long-range effects - an ubiquitous feature of nonequilibrium states - show up also in the nature of stabilization.
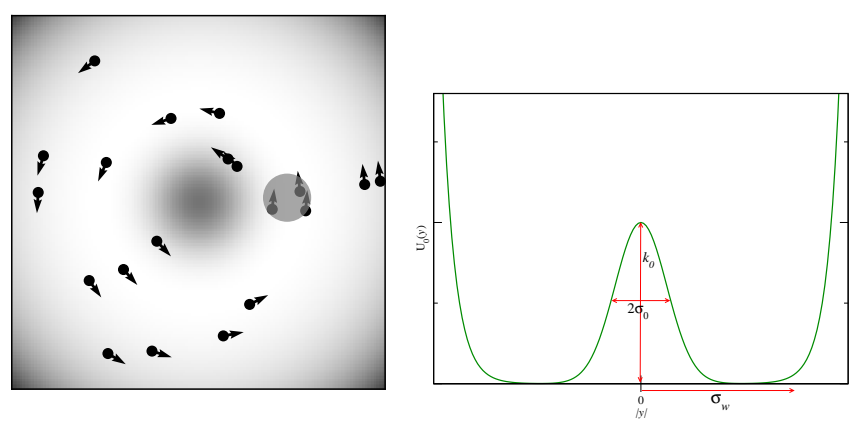

Fig. 1: An illustration of the class of systems considered. Left: The gray background depicts the confining potential, darker regions corresponding to higher values of the potential. The black dots represent the particles of the active medium, with an arrow in the direction of the flow. The shaded circle represents the probe. Right: Parameters of the Mexican hat potential $V(r)$.

We start by giving the general coupled dynamics of medium and probe and define the statistical forces and the corresponding stiffness in the limit of a quasi-static probe. We demonstrate the stabilization effect of the nonequilibrium driving for different types of differential rotation and potentials. We also state a sufficient condition under which we are able to prove rigorously the positivity of the (nonequilibrium) Lamb shift. Finally we employ numerics to explore the stabilization beyond the quasi-static regime. More technical derivations are collected in an Appendix.

Statistical force and stiffness. - We consider a two-dimensional system in which $N$ driven particles and a probe move in a thermal environment at temperature $T$, idealized here by using an overdamped Langevin dynamics. We refer to the driven particles at positions $y^{i}$ as the medium, which are mutually noninteracting, as an ideal gas, but are subject to a sufficiently confining effective potential $V(y)$. Each interacts with the probe via the potential $U_{I}(|x-y|)$ depending on the distance to the probe at position $x ; U(x, y)=V(y)+U_{I}(|x-y|)$ is the total potential. We use the notation $\nabla U$ for the gradient with respect to the medium particle position $y$ and $\nabla_{x} U$ is the gradient with respect to the probe position $x$. Furthermore, each of the medium particles is subject to a solenoidal driving force $F(y)$ which means that $\nabla \cdot F=0$ and $F$ is not derivable from a potential. The mobility for the medium particles is denoted by $\chi>0$ and the damp- ing coefficient for the probe is $\gamma>0$, so that the joint dynamics becomes, for $i=1, \ldots, N$,

$$
\begin{aligned}
\dot{y}_{t}^{i} & =\chi\left[F\left(y_{t}^{i}\right)-\nabla U\left(x_{t}, y_{t}^{i}\right)\right]+\sqrt{2 \chi T} \xi_{t}^{i}, \\
\gamma \dot{x}_{t} & =-\sum_{i=1}^{N} \nabla_{x} U_{I}\left(\left|x_{t}-y_{t}^{i}\right|\right)+\sqrt{2 \gamma T} \xi_{t}
\end{aligned}
$$

all smoothly depending on the positions and under free boundary conditions at infinity. The $\xi_{t}^{i}, \xi_{t}$ are independent standard white noises. Later for convenient simulation we apply also an extra confining potential $V_{p}$ on the probe.

For simplicity of the argument, we assume that the origin is a special point of symmetry, in the sense that $F \perp \nabla U\left(x=0, y^{i}\right)$ meaning that the driving is always orthogonal to the force on the medium particles when $x=0$ (probe is at the origin). An example is provided by particles in a rotation symmetric external potential $V\left(y^{i}\right)=V\left(\left|y^{i}\right|\right)$ which are driven by a rotational driving force $F$ having only an angular (and no radial) component around the origin.

The quasi-static regime for the probe is reached when the medium has a very small relaxation time compared to the probe, or $\gamma \chi \rightarrow \infty$. (Below we also explore the joint dynamics when the time-scale separation between (fast) medium and (slow) probe is not infinite.) The averaged mechanical effect of the medium particles on the probe is described by the statistical force

$$
\begin{aligned}
f(x) & =-\int \prod_{i=1}^{N}\left[\mathrm{~d} y^{i} \rho_{x}\left(y^{i}\right)\right] \sum_{i=1}^{N} \nabla_{x} U\left(x, y^{i}\right) \\
& =-N \int \mathrm{d} y \rho_{x}(y) \nabla_{x} U(x, y) \\
& =-N\left\langle\nabla_{x} U_{x}\right\rangle^{x}
\end{aligned}
$$

where we average over the stationary medium density $\rho_{x}(y)$ for the dynamics of a single driven particle while keeping the probe at position $x$. We also write $U_{x}(y)=$ $U(x, y)$ and $\langle\cdot\rangle^{x}$ is the expectation over $\rho_{x}$. We always have the origin to be a fixed point in the sense that the statistical force $f(x=0)=0$ vanishes there. The statistical force has components $f=\left(f_{1}, f_{2}\right)$ depending on the decomposition in orthogonal coordinates.

To investigate the stability of the probe near the origin, we introduce the stiffness matrix at a fixed point $x$

$$
M_{j k}(x)=-\frac{\partial f_{k}}{\partial x_{j}}
$$

A sufficient condition for local stability 9 is the positivity of the symmetric part of the corresponding stiffness matrix (4). For $x=0$ the eigenvalue in the radial direction provides the effective spring constant by which the probe is attracted to the origin.

Under equilibrium, i.e., for $F=0$ in (2), the statistical force $f(x)=T N \nabla_{x} \log \mathcal{Z}_{x}$ derives from the free energy, where the (single-particle) partition function $\mathcal{Z}_{x}$ is

$$
\mathcal{Z}_{x}=\int \mathrm{d} y \exp \left[-\beta U_{x}(y)\right]
$$


for $\beta=1 / T$. The equilibrium $(F=0)$ stiffness is

$$
M_{j k}^{\mathrm{eq}}(x)=T \frac{\partial^{2} \log \mathcal{Z}_{x}}{\partial x_{j} \partial x_{k}}
$$

which is automatically symmetric, but will here be a negative matrix for $x=0$ implying that the probe is unstable at the origin under equilibrium conditions.

The main subject of the paper is to show how for the probe the origin stiffens under nonequilibrium. We will give a formula for the symmetric part of the stiffness matrix including the nonequilibrium Lamb shift. Apart from the simulation and exact numerical evaluation of that formula we also state a specific sufficient condition for improved stability upon driving.

Nonequilibrium stabilization. - We consider the ideal-gas model of the driven medium (1) which we now write as a single-particle diffusion with $\chi=1$,

$$
\dot{y}_{t}=F\left(y_{t}\right)-\nabla U_{x}\left(y_{t}\right)+(2 T)^{1 / 2} \xi_{t}, \quad \nabla \cdot F=0
$$

(Remember that $\nabla$ is the gradient on the $y$-variable.) The potential and driving field are

$$
U_{x}(y)=V(|y|)+U_{I}(|y-x|), \quad F(y)=\varepsilon|y| \omega(|y|) \hat{e}_{\varphi}
$$

where in polar coordinates $(r, \varphi)$ we have a radial rotation profile $\omega(r)$ and the driving is in the angular direction (with unit vector $\hat{e}_{\varphi}$ ). The backward generator of that driven diffusion equals

$$
L_{x}=\left(F-\nabla U_{x}\right) \cdot \nabla+T \Delta=L_{x}^{\mathrm{eq}}+\Omega
$$

with $\Omega=F \cdot \nabla=\varepsilon \omega(r) \partial / \partial \varphi$ being the flow under the rotational driving force.

To be specific we take here the external potential

$$
V(r)=k_{0} e^{-\frac{r^{2}}{2 \sigma_{0}^{2}}}+e^{r-\sigma_{w}}
$$

which is a Mexican hat with outer radius $\sigma_{w}$ and with the top at the origin of size $\sigma_{0}$ and height $k_{0}$; see also Fig 1 (b). For the local interaction we take

$$
U_{I}(x, y)=-\lambda\left[1-\frac{(x-y)^{2}}{\sigma^{2}}\right]^{2}
$$

with a cut-off at $|x-y|=\sigma$. Fig. 2 (a) shows the dependence on $\varepsilon$ of the stiffness (4) (or effective spring constant) $m=M_{11}=M_{22}$ at $x=0$, as obtained from quasi-static numerical simulations, for various choices of rotation profiles $\omega(r)$. We observe that the Lamb shift is always positive and the origin eventually becomes stable as the driving is increased; there are no dramatic differences between the types of rotation, even including the case $\omega(r)=1 / \sqrt{r}$ where the angular speed is decreasing radially outward. We have explored a number of other potentials but making sure always that the equilibrium case shows an unstable origin; the same effect of stabilization by rotation is seen in all the cases. Fig. 2 (b) shows the temperature dependence for the constant rotation profile $\omega(r)=1$ showing that the threshold value for $\varepsilon$ depends very little on $T$. We now give the theory leading to and explaining these findings.

The stiffness (4) per medium particle (or for $N=1$ ) is

$$
\begin{aligned}
M_{j k}(x) & =\frac{\partial}{\partial x_{j}} \int \mathrm{d} y \rho_{x}(y) \frac{\partial U_{x}}{\partial x_{k}}(y) \\
& =\left\langle\frac{\partial^{2} U_{x}}{\partial x_{j} \partial x_{k}}\right\rangle^{x}+\left\langle\frac{\partial \log \rho_{x}}{\partial x_{j}} \frac{\partial U_{x}}{\partial x_{k}}\right\rangle^{x}
\end{aligned}
$$

To make the last term more explicit we need to find a useful expression for the response of the stationary distribution $\rho_{x} \rightarrow \rho_{x+\mathrm{d} x}$ under a change in probe position. That can be obtained from the linear response theory around steady nonequilibrium [10. In the Appendix we derive the response formula yielding the stiffness at the origin $M_{j k}=M_{j k}(0)$,

$$
\begin{aligned}
M_{j k}= & \left\langle\left.\frac{\partial^{2} U_{x}}{\partial x_{j} \partial x_{k}}\right|_{0}\right\rangle^{0}-\beta\left\langle\left.\frac{\partial U_{x}}{\partial x_{j}}\right|_{0} ;\left.\frac{\partial U_{x}}{\partial x_{k}}\right|_{0}\right\rangle^{0} \\
& -\beta\left\langle\left.\left.\Omega \frac{\partial U_{x}}{\partial x_{j}}\right|_{0} \frac{1}{L_{0}} \frac{\partial U_{x}}{\partial x_{k}}\right|_{0}\right\rangle^{0}
\end{aligned}
$$

The first line indeed equals the equilibrium form of (5): it subtracts from the intrinsic mechanical stiffness (first term) the fluctuation part (in the covariance $\langle u ; v\rangle=$ $\langle u v\rangle-\langle u\rangle\langle v\rangle)$ which can easily make that first line (the equilibrium stiffness) a negative matrix. The second line of (12) contains the "frenetic" contribution as it involves the non-entropic response to the change in density $\rho_{x}$ as needed for (11). The backward generator is defined in (8). One of our results, explained in the Appendix, is to use (12) to provide an exact numerical algorithm to obtain the Lamb shift.

As an immediate application we use formula (12) to rigorously prove the positivity of the nonequilibrium Lamb shift for $\omega(r)=1$ (rigid rotation). In that case $\Omega$ simplifies to $\tilde{\Omega}=\varepsilon \partial / \partial \varphi$ for which

$$
\left[L_{0}^{\mathrm{eq}}, \tilde{\Omega}\right]=\left[L_{0}, \tilde{\Omega}\right]=0
$$

and we also have the antisymmetry, $\tilde{\Omega}^{*}=-\tilde{\Omega}$ as proven in the Appendix. Therefore $\tilde{\Omega}$ generates a one-parameter symmetry of the equilibrium dynamics for $x=0$ and the symmetric part of $\tilde{\Omega} L_{0}^{-1}$ is a positive operator as is obvious from rewriting it as

$$
\begin{aligned}
& \frac{1}{2}\left[\frac{\tilde{\Omega}}{L_{0}}+\left(\frac{\tilde{\Omega}}{L_{0}}\right)^{*}\right]=\frac{1}{2}\left[\frac{\tilde{\Omega}}{L_{0}}-\frac{\tilde{\Omega}}{L_{0}^{*}}\right] \\
& =-\frac{\tilde{\Omega}^{2}}{L_{0} L_{0}^{*}}=\left(\frac{\tilde{\Omega}}{L_{0}}\right)\left(\frac{\tilde{\Omega}}{L_{0}}\right)^{*} \geq 0
\end{aligned}
$$

Substituting into 12 , the symmetric part of the stiffness matrix at the origin, $M_{j k}^{(s)}=\left[M_{j k}+M_{k j}\right] / 2$, obtains the 
form

$$
M_{j k}^{(s)}=M_{j k}^{\mathrm{eq}}+\beta\left\langle\left.\frac{\tilde{\Omega}}{L_{0}} \frac{\partial U_{x}}{\partial x_{j}}\right|_{0} ;\left.\frac{\tilde{\Omega}}{L_{0}} \frac{\partial U_{x}}{\partial x_{k}}\right|_{0}\right\rangle^{0}
$$

We conclude that the nonequilibrium Lamb shift as given by the second term is a positive matrix, symmetric with respect to the driving reversal. Since that Lamb shift is of second order for small $\varepsilon$, the effect described here falls outside the usual linear response regime around equilibrium and is beyond the standard treatments via irreversible thermodynamics. On the other hand, for a strong driving the nonequilibrium rotation appears to reshape the second term of the equilibrium line in $(12)$, canceling it completely for $\varepsilon \rightarrow \infty$. In this limit we find

$$
M_{j k}^{(s)} \rightarrow\left\langle\left.\frac{\partial^{2} U_{x}}{\partial x_{j} \partial x_{k}}\right|_{0}\right\rangle^{0}
$$

which reduces the stiffness to a thermal average of the intrinsic or mechanical stiffness only. Positivity of that mechanical stiffness combined with the negativity of the equilibrium stiffness signals the transition in $\varepsilon$ between an unstable and a stable origin for the probe. Observe furthermore that the Lamb shift in (14) can be interpreted as a covariance of time-integrated excess work because

$$
\left.\frac{\tilde{\Omega}}{L_{0}} \nabla_{x} U_{x}(y)\right|_{0}=\int_{0}^{+\infty} \mathrm{d} t\left\langle g_{0}\left(y_{t}\right) \mid y_{t=0}=y\right\rangle^{0}
$$

where $g_{x}=\nabla_{x} F \cdot\left(F-\nabla U_{x}\right)$ is the gradient of the mean instantaneous power of the driving force; see, e.g., Appendix $\mathrm{A}$ in [7]. Finally we note that even for local interactions $U_{I}$ and intermediate values of $\varepsilon$ we can expect a rather strong dependence in the Lamb shift on the medium density far away from the origin. The reason is that $L_{0}^{-1}$, just like the Green function of the Laplacian, generally has logarithmic (in two dimensions) or algebraic (in three dimensions) asymptotics.

To make that point of nonlocality clear we give in Fig. 3 the dependence of the stiffness $m$ on changes in the external potential far away from the origin. That is done by adding a local hill to the potential (9) at a distance $r_{b}>\sigma_{0}$ from the origin,

$$
\tilde{V}(r)=V(r)+k_{b} e^{-\frac{\left(r-r_{b}\right)^{2}}{2 \sigma_{b}^{2}}}
$$

The stiffness at the origin starts to depend on the hill strength $k_{b}$ as $\varepsilon$ is increased, as can be seen from Fig. 3 . That nonlocal dependence of the Lamb shift $\Delta m=m-$ $m^{\text {eq }}$ on far-away features is another manifestation of the generic long range correlations in nonequilibrium systems.

Qualitatively, however, a local linearization shows exactly the same stabilization phenomenon. A linear example consists of a rotation-symmetric quadratic potentials $V(y)=\kappa y^{2} / 2, \quad U_{I}(|y-x|)=\lambda(y-x)^{2} / 2 \quad(\kappa+\lambda>0)$ for
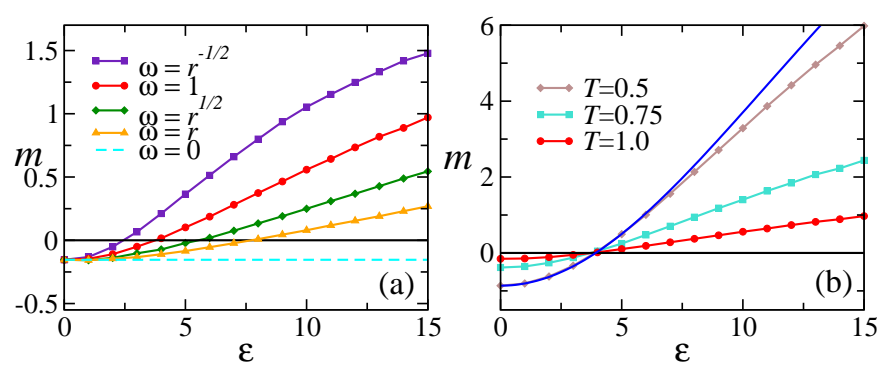

Fig. 2: (a) Stiffness of the statistical force at the origin for various rotation profiles as function of the driving $\varepsilon$. Parameters: $T=1, \lambda=5, k_{0}=1, \sigma=1$ and $\sigma_{0}=1$. (b) Stiffness of the statistical force at the origin for $\omega(r)=1$ with driving $\varepsilon$ for various temperatures. The solid line shows the stiffness corresponding to the linearized potential following (18).

which the equilibrium stiffness (23) is $m^{\mathrm{eq}}=\lambda \kappa /(\kappa+\lambda)$ and the Lamb shift equals

$$
\Delta m=\frac{\varepsilon^{2} \lambda^{2}}{(\kappa+\lambda)\left[(\kappa+\lambda)^{2}+\varepsilon^{2}\right]}
$$

in accord with the results in ref. 7]. For $0>\kappa>-\lambda$ the threshold driving amplitude equals $\varepsilon_{c}=\sqrt{-\kappa(\kappa+\lambda)}$ such that $m<0$ (instability) for $|\varepsilon|<\varepsilon_{c}$ whereas $m>0$ (stability) for $|\varepsilon|>\varepsilon_{c}$. Note that here neither the stiffness nor the threshold depends on temperature $T$. For the nonlinear potentials $(9)-10$ we can approximately restore the linear behavior at low enough temperatures, e.g., under the assumption that the total potential $U(x=0, y)$ for the medium particles exhibits a global minimum at the origin $y=0$. In that regime the probe stiffness is dominated by those particles which have been trapped around the origin and the linearization is in order. This is explored in Fig. 2(b) where the solid line shows the plot of $m$ following (18) with effective parameters $\lambda_{\text {eff }}=4 \lambda / \sigma^{2}=20$ and $\kappa_{\mathrm{eff}}=-k_{0} / \sigma_{0}^{2}=-1$; the agreement is quite good for small $\varepsilon$, covering the range when the probe becomes stable.

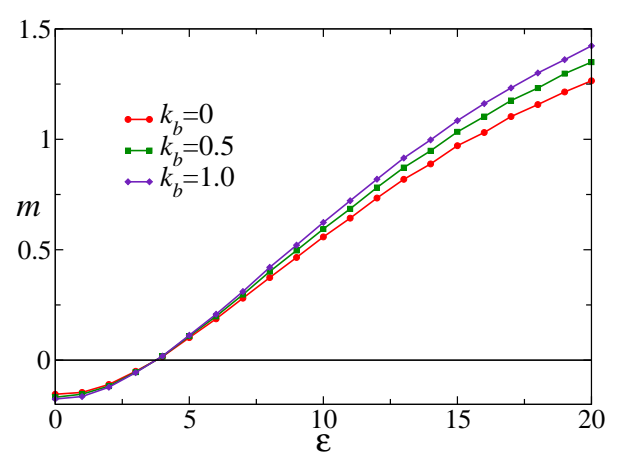

Fig. 3: The long-range dependence of the stiffnes $m$ on $k_{b}$ in the external potential $\tilde{V}(r)$ for large enough $\varepsilon$. We took $r_{b}=4, \sigma_{b}=0.3$ and temperature $T=1.0$.

Beyond the quasi-static limit. - We return to the coupled system of equations (1)-(2) but now for finite $\gamma \chi$. 
Away from the quasi-static regime we can still look at the total force on the probe in the joint (medium plus probe) steady ensemble. We then consider the conditional expectation

$$
f(X)=-N\left\langle\nabla_{x} U(x, y) \mid x=X\right\rangle
$$

which in the quasi-static limit or in equilibrium coincides with (3). Note that in nonequilibrium there is no reason for the force $f$ to be derived from the effective potential $V_{\text {eff }}(X)=-T \log \langle\delta(x-X)\rangle$.

We performed simulations of Eqs. (1)-(2) with the stochastic Runge-Kutta algorithm [11. The external and the interaction potentials remain the same as in $(9)$ and (10). The driving is via rotation field $F(y)=\varepsilon|y| \hat{e}_{\varphi}$. As we are interested in studying the stability of the origin and to avoid trajectories in which the probe exits the bath region, the probe is confined via the external potential

$$
V_{p}(x)=e^{|x|-\left(\sigma_{w}+1\right)}
$$
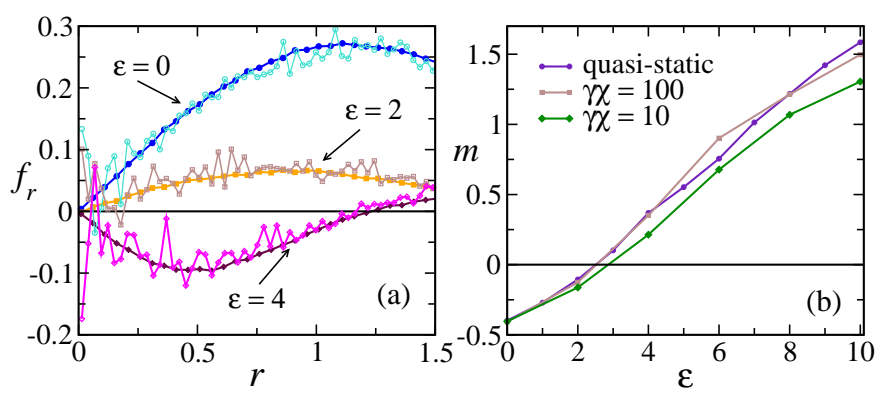

Fig. 4: (a) The radial statistical force on the quasi-static probe under nonequilibrium. Results for quasi-static simulations and joint simulations (with $\gamma \chi=100$ ) are superimposed, the latter displaying stronger fluctuations. The variation of $\varepsilon$ allows us to see the transition from unstable to stable for the fixed point at the origin. (b) The stiffness as a function of $\varepsilon$ for both quasi-static and full $(\gamma \chi=100$ and 10) are superimposed.

The parameters are $T=1, \chi=1, \sigma_{w}=6, \sigma_{0}=1, k_{0}=$ $1 / 2, \sigma=1, \lambda=2$ and $\gamma=10$ or $\gamma=100$, with higher mobility for $\gamma=10$. The results remain the same qualitatively for other values of the parameters, e.g., for much shorter radius $\sigma$ of interaction between the probe and the medium particles but then we need to take larger $\varepsilon$. A direct comparison between quasi-static and joint simulations, for the radial force on the probe, is shown in Fig. 4 (a) where good agreement is found. The results beyond the quasi-static limit show of course more fluctuations, related to the sampling when the probe is moving, with lower radii being most affected.

The $\varepsilon$ dependence of the stiffness, shown in Fig. 4 (b) confirms the agreement with our quasi-static results. Increasing the probe mobility, we can observe that the behaviour of the stiffness changes and deviates from the quasi-static result, for increased values of the driving $\varepsilon$.
In equilibrium, i.e., for $\varepsilon=0$, we observe no deviation at all, as expected.

As the probe now moves around, we get access also to its stationary probability density. In Fig. 5 (a) we observe a dip in the radial density $P(r)$ for $\varepsilon=0$ that is typical to an unstable fixed point. Increasing $\varepsilon$ leads to a transformation of this dip into a flat density (for near zero stiffness) and then a local excess of density (for a stiff origin). There is no concurrent change in the local radial density of bath particles $\rho(r)$ when integrating out the probe position; $\rho(r)$ is displayed in Fig. 5 (b), the nonequilibrium driving does not change its shape. It also means that from just observing the medium radial profile, we cannot infer the probe's attraction or repulsion from the origin.
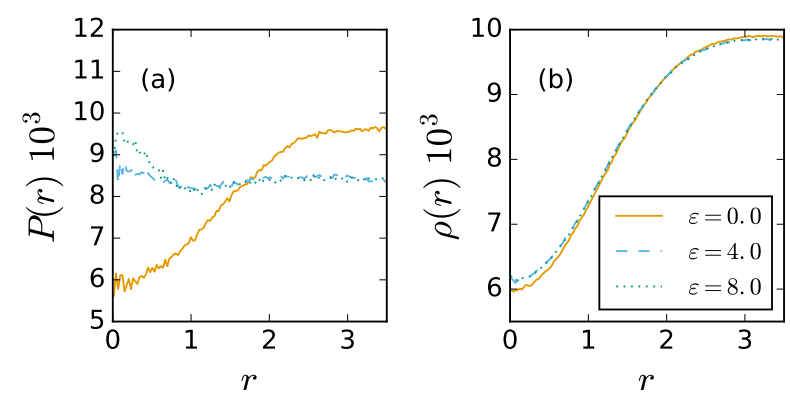

Fig. 5: (a) The radial distribution $P(r)$ of the probe for joint simulations of Eqs. (1)-(2). (b) The radial distribution $\rho(r)$ of the medium for the same simulation set.

Conclusion. - In a specific nonequilibrium environment different shapes, phases or configurations may become more stable than in equilibrium. A systematic treatment uses nonequilibrium response theory to evaluate the stiffness or effective spring constant near a fixed point for the mean force on the probe. There appears a simple sufficient condition for increased stability in terms of the invariance of the equilibrium dynamics under the driving flow, but our examples showed great robustness of the stabilization for all types of differential rotation and beyond the quasi-static limit. A first, more physical, explanation of the results can be obtained by linearising the problem locally around the origin, where a simple calculation yields the desired effect of improved stability. However, we have noted important long-range effects due to the nonequilibrium nature of the problem, at least beyond the regime of low temperatures where the local linear approximations around the origin often provide a valid approximation. We found at least in the case of rigid rotation $\omega(r)=1$ that the nonequilibrium stiffness becomes equal to the intrinsic stiffness of the medium-probe coupling as the driving gets very high; see 15). The effect therefore seems to be mechanical, with the driving eliminating the force fluctuations which make the origin unstable in the equilibrium case.

Yet we must realize that the Lamb shift in the effective spring constant is second order in the nonequilibrium 
driving and thus the effect falls outside equilibrium-like energy-entropy considerations. That is finally the most interesting part theoretically, as we enter here the problem of stabilisation outside the usual thermodynamic framework, where we believe much needs to be discovered and analyzed. The strategy that we have followed here of using a class of models with a common geometry but different interactions and rotational profiles is expected to be relevant to other situations, such as shear flows for instance, to provide statistical mechanical treatments of probe-focussing in fluid flow.

$$
* * *
$$

We thank Alexandre Lazarescu for helpful discussions. $\mathrm{PdB}$ is a postdoctoral fellow of the Research FoundationFlanders (FWO). UB acknowledges the financial support by the ERC under Starting Grant 279391 EDEQS.

\section{Appendix. -}

Steady medium at $x=0$. The case $x=0$ is particularly convenient because of the orthogonality relation

$$
F \cdot \nabla U_{0}=0
$$

easily derived as we have assumed that the external potential is purely radial. As also $\nabla \cdot F=0$ we conclude that $\rho_{0}$ is invariant for $\Omega=\varepsilon \omega(r) \partial_{\varphi}$ and as a consequence $\rho_{0}$ equals the equilibrium distribution $\rho_{0} \propto \exp \left[-\beta U_{0}(r)\right]$ for all $\varepsilon$.

Note also that for all $\omega(r)$ and arbitrary functions $u$ and $v$,

$$
\begin{aligned}
\langle u \Omega v\rangle^{0} & =\int \rho_{0} u \nabla \cdot(F v)=\varepsilon \int \rho_{0} u \omega \partial_{\varphi} v \\
& =-\varepsilon \int \rho_{0} v \omega \partial_{\varphi} u=-\langle v \Omega u\rangle^{0}
\end{aligned}
$$

i.e., $\Omega$ is an antisymmetric operator, $\Omega^{*}=-\Omega$, while by detailed balance $L_{0}^{\mathrm{eq}}$ is symmetric. This means that the (driven) adjoint dynamics for $x=0$ has the generator $L_{0}^{*}=L_{0}^{\mathrm{eq}}-\Omega$ which differs from $L_{0}$ only by the driving reversal. Therefore 13 ensures the normality property, $\left[L_{0}, L_{0}^{*}\right]=0$.

Response formula. Stationarity implies that $\left\langle L_{x} u\right\rangle^{x}=$ 0 which we can differentiate at $x=0$ to produce

$$
\begin{aligned}
0 & =\left\langle\left(\partial_{j} \log \rho_{0} L_{0}+\partial_{j} L_{0}\right) u\right\rangle^{0} \\
& =\left\langle u L_{0}^{*} \partial_{j} \log \rho_{0}-\nabla \partial_{j} U_{0} \cdot \nabla u\right\rangle^{0} \\
& =\left\langle u\left\{L_{0}^{*} \partial_{j} \log \rho_{0}-\beta \nabla U_{0} \cdot \nabla \partial_{j} U_{0}+\Delta \partial_{j} U_{0}\right\}\right\rangle^{0} \\
& =\left\langle u\left\{L_{0}^{*} \partial_{j} \log \rho_{0}+\beta L_{0}^{\mathrm{eq}} \partial_{j} U_{0}\right\}\right\rangle^{0}
\end{aligned}
$$

That yields the equation $L_{0}^{*} \partial_{j} \log \rho_{0}=-\beta L_{0}^{\mathrm{eq}} \partial_{j} U_{0}$. We have used the simplified notation $\partial_{j}=\partial / \partial x_{j}, \partial_{j} v_{0}=$ $\left.\left(\partial v / \partial x_{j}\right)\right|_{x=0}$ for all functions $v(x)$. Finally, from the previously derived $L_{0}^{*}=L_{0}-\Omega$ for the adjoint of $L_{0}$ under $\rho_{0}$, that implies

$$
L_{0}^{*}\left(\partial_{j} \log \rho_{0}+\beta \partial_{j} U_{0}\right)=-\beta \Omega \partial_{j} U_{0}
$$

the solution of which is the response formula

$$
\beta^{-1} \partial_{j} \log \rho_{0}=-\partial_{j} U_{0}+\left\langle\partial_{j} U_{0}\right\rangle^{0}-\left(L_{0}^{*}\right)^{-1} \Omega\left(\partial_{j} U_{0}\right)
$$

Note that the driving $\varepsilon$ does not at all have to be small as we are not doing linear response in $F$ but in $x$ (for fixed driving $F$ ).

The formula (21) immediately gives rise to formula 12 by substituting it into (11). To go from there to 14 for the case of $\omega(r)=1$, we need the commutativity (13), $\left[L_{0}, \tilde{\Omega}\right]=0$ with $\tilde{\Omega}=\varepsilon \partial_{\varphi}$ and

$$
L_{0}=\varepsilon \frac{\partial}{\partial \varphi}-U_{0}^{\prime} \frac{\partial}{\partial r}+T\left[\frac{1}{r} \frac{\partial}{\partial r}\left(r \frac{\partial}{\partial r}\right)+\frac{1}{r^{2}} \frac{\partial^{2}}{\partial \varphi^{2}}\right]
$$

In general, for $\Omega=\omega(r) \tilde{\Omega},\left[L_{0}, \Omega\right] \neq 0$ and only the orthogonality $F \cdot \nabla U_{0}=0$ remains verified.

Stability. Asymptotic stability is equivalent to the strict positivity of the real parts of all eigenvalues of $M$ $\left(\Rightarrow\right.$ strict contractivity of the semigroup $e^{-M t}$ ). It suffices in general to have a simple Lyapunov function. See for example Theorem 3.6 in ref. [9].

The simplest candidate for a Lyapunov function is $\lambda(x)=|x|^{2}$. If the induced probe dynamics is overdamped, $\gamma \dot{x}_{t}=f\left(x_{t}\right)$ with some friction $\gamma>0$ and linear approximation $f(x)=-M x$, then

$$
\gamma \frac{\mathrm{d} \lambda\left(x_{t}\right)}{\mathrm{d} t}=-2 x_{t} \cdot M_{s} x_{t}
$$

and hence $\lambda(x)$ is (exponentially) Lyapunov with attractor at $x=0$ if and only if $M_{s}>0$, i.e., if all its eigenvalues are strictly positive. Provided that is verified, then the antisymmetric part $M_{a}=\left(M-M^{\dagger}\right) / 2$ representing rotational forces does essentially not matter for stability (though it of course enters the phase portrait). If $M$ is a normal matrix, $\left[M, M^{\dagger}\right]=0=\left[M_{s}, M_{a}\right]$, then the real parts of eigenvalues of $M$ coincide with the eigenvalues of $M_{s}$. In that case, asymptotic stability is equivalent to $M_{s}>0$.

Computational algorithm for the Lamb shift. Let us next give an algorithm to find these Lamb shifts via the solution of certain differential equations.

By rotation symmetry the most general form of the stiffness matrix (4) for the probe around $x=0$ is

$$
M=\left(\begin{array}{cc}
m & -a \\
a & m
\end{array}\right)
$$

At equilibrium $a^{\mathrm{eq}}=0$, and

$$
m^{\mathrm{eq}}=\frac{\pi \beta}{\mathcal{Z}_{0}} \int_{0}^{\infty} V^{\prime} U_{I}^{\prime} e^{-\beta\left(V+U_{I}\right)} r \mathrm{~d} r, \quad{ }^{\prime}=\frac{\partial}{\partial r}
$$

When $\varepsilon>0$ and for angular rotation $\omega(r)$ we get $m=$ $m^{\text {eq }}+\Delta m$. We show here that the Lamb shift can be expressed as

$$
\Delta m=\frac{1}{2} \varepsilon \beta \operatorname{Im}\left\langle U_{I}^{\prime} \omega \Psi\right\rangle^{0}
$$


where $\Psi(r)$ solves the differential equation

$$
-U_{0}^{\prime} \Psi^{\prime}+\frac{T}{r}\left(r \Psi^{\prime}\right)^{\prime}-\frac{T}{r^{2}} \Psi+i \varepsilon \omega(r) \Psi=-U_{I}^{\prime}
$$

We first note that formula 12 can be written as

$$
\Delta M_{j k}=\left\langle\left(\omega \nabla_{j} U_{I}\right) \frac{1}{L_{0}^{\mathrm{eq}}+\omega \tilde{\Omega}} \tilde{\Omega}\left(\nabla_{k} U_{I}\right)\right\rangle^{0}
$$

For the rightmost vector we need, in Cartesian coordinates,

$$
\frac{\partial}{\partial \varphi}\left(\nabla U_{I}\right)=U_{I}^{\prime}(r)(-\sin \varphi, \cos \varphi)
$$

and we want to find $h(y)=\left(h_{1}(y), h_{2}(y)\right)$ with $\langle h\rangle^{0}=0$ so that $\left(L_{0}^{\mathrm{eq}}+\omega \tilde{\Omega}\right) h=-\varepsilon U_{I}^{\prime}(\sin \varphi,-\cos \varphi)$. Going to complex notation, we write $h(y)=\Psi(r) e^{i \varphi}$ and note that for any $\Phi=\Phi(r)$,

$$
\begin{aligned}
L_{0}^{\mathrm{eq}}\left(\Phi e^{i \varphi}\right) & =(\Lambda \Phi) e^{i \varphi} \quad \text { where } \\
\Lambda \Phi & =-U_{0}^{\prime} \Phi^{\prime}+\frac{T}{r}\left(r \Phi^{\prime}\right)^{\prime}-\frac{T}{r^{2}} \Phi
\end{aligned}
$$

As a consequence,

$$
\frac{1}{L_{0}} \tilde{\Omega}\left(\nabla U_{I}\right)=\varepsilon(\operatorname{Im},-\operatorname{Re})\left(\Psi e^{i \varphi}\right)
$$

where $\Psi,\left\langle|\Psi|^{2}\right\rangle^{0}<+\infty$ solves

$$
(\Lambda+i \varepsilon \omega) \Psi=-U_{I}^{\prime}
$$

Combining with $\nabla U_{I}=(\operatorname{Re}, \operatorname{Im})\left(U_{I}^{\prime} e^{i \varphi}\right)$ it finally yields (24).

Since $\operatorname{Im} \Psi=O(\varepsilon)$, the Lamb shift is $O\left(\varepsilon^{2}\right)$ as expected due to the symmetry $\varepsilon \leftrightarrow-\varepsilon$. The differential equation 25) is solved with the routine NDSolve of Mathematica. The density of the medium is fixed at the boundary of the system as $\bar{\rho}(R)=1$.

In the case where $\omega(r)=1$ we can take $\tilde{\Psi}$ solving

$$
(\Lambda+i \varepsilon) \tilde{\Psi}=-U_{I}^{\prime}
$$

and the Lamb shift obtains the simplified expression

$$
\Delta m=\frac{1}{2} \varepsilon^{2} \beta\left\langle|\tilde{\Psi}|^{2}\right\rangle^{0}
$$

giving an alternative to 14 . As an example, for the linear case (18), equation 29) for $\omega=1$ has the solution $\tilde{\Psi}(r)=$ $\lambda r /(\kappa+\lambda-i \varepsilon)$.

\section{REFERENCES}

[1] Kapitza P. L., Dynamical stability of a pendulum when its point of suspension vibrates in Collected papers of $P$. L. Kapitza, edited by Ter HAAR D., Vol. II (Pergamon Press) 1965 pp. $714-725$.

[2] Krieger M. S., arXiv, (2015) 1512.04423.

[3] Marchetti M. C., Joanny J. F., Ramaswamy S., Liverpool T. B., Prost J., Rao M. and Simha R. A., Rev. Mod. Phys., 85 (2013) 1143.
[4] Barré J., Bouchet F., Dauxois T. and Ruffo S., Phys. Rev. Lett., 89 (2002) 110601.

[5] Sheshik R., Recho P. and Truskinovsky L., arXiv, (2015) 1512.04423.

[6] Basu U., Maes C. and NetočnÝ K., Phys. Rev. Lett., 114 (2015) 250601.

[7] Basu U., Maes C. and NetočnÝ K., New J. Phys., 17 (2015) 115006.

[8] Breuer H. P. and Petruccione F., The Theory of Open Quantum Systems (Oxford University Press, Oxford) 2002.

[9] Khalil H. K., Nonlinear Systems (Prentice Hall) 2002.

[10] Baiesi M., Maes C. and Wynants B., J. Stat. Phys., 137 (2009) 1094.

[11] Brańka A. C. and Heyes D. M., Phys. Rev. E, 58 (1998) 2611. 